\title{
Disabling disability claims in gastroenterology
}

\author{
Jack Richman $\mathrm{MD}^{1}$, Paul Adams MD²
}

$\mathrm{D}_{\mathrm{D}}^{\mathrm{r}}$ Jack Richman is the Executive Vice President and Medical Director of ASSESSMED. Dr Richman, after being in family practice from 1968 to 1977 , specialized in occupational medicine, pain management and disability evaluation. He is Past President of the Canadian Society of Medical Evaluators. In addition, Dr Richman is a Diplomat of the American Academy of Pain Management, and a founding Member of the Canadian Pain Society and the International Association for the Study of Pain.

PA: Can you give us some background information on the assessment of disability in Canadians?

JR: While insurance companies may make it seem that we are covered for any and all illnesses and injuries from cradle to grave, that is not the case. The role of insurance traditionally is to provide benefits for income replacement in terminally ill cases, or during a transition period from an acute illness to functional improvement.

Once management of the acute condition or the acute phase of a chronic condition has occurred, one no longer medically qualifies. Unfortunately, this is not what insurance companies convey when they sell their policies. We, and the patients, often take away the impression that we receive benefits until we are cured or symptom-free. However, the presence of symptoms or perceived functional difficulty does not mean our patients qualify for benefits. For example, after a colectomy, ulcerative colitis patients are cured and usually return to work. This is often not true of Crohn's disease. Two different inflammatory bowel diseases, two different patterns and outcomes.

To automatically qualify, a patient must have a bona fide and well-defined medical condition that is in an acute or progressive state. Symptoms alone do not qualify the patient for benefits. The American Medical Association's Guides to the Evaluation of Permanent Impairment, 4th Edition (1), states that disability is due to a "medically determinable" impairment. This is defined as an impairment that has demonstrable anatomic, physiological or psychological abnormalities. Abnormalities that manifest themselves only as symptoms do not qualify.

PA: So when does a patient qualify for disability?

JR: In my opinion and experience what I see is either:

1. The condition must be one that has well-defined pathology and is terminal (eg, cancer with metastatic disease), and/or progressive (eg, multiple sclerosis), or of such an acute severity that there can be no improvement (eg, severely traumatic brain injured persons), or is progressive (eg, uncontrolled diabetes with its sequelae of kidney failure), or

2. The condition must be one that has well-defined pathology (eg, by testing such as an electromyogram or biopsy), has intermittent exacerbations that prevent one from functioning on a regular basis (eg, Parkinson's disease, unstable Crohn's disease, unstable diabetes, unstable epilepsy or unstable colitis). However, ulcerative colitis can be cured with a colectomy.

Irritable bowel syndrome does not qualify despite the significant symptoms. Most insurers do not accept syndromes because they have no known etiological agent, no well-defined pathology and there is no test that is positive and specific to that condition. Any finding is the result of the condition and not the cause of it. Causes may be treatable and/or preventable and, as such, are the reasons why the insurers look to physicians to find an etiological agent or well-defined pathology, if it is present. Once found, we can then determine if it is treatable. If there is no cause of the symptoms or syndrome, then anyone with a set of symptoms can self-diagnose and, therefore, physicians are not required. We only become form fillers. PA: We have a huge number of patients in gastroenterology that are demanding chronic disability status. We have a barrage of forms that are coming our way and a growing hostility among patients, families and physicians about this situation. I would like to draw on your experience in the general field of disability claims to guide us in gastroenterology practice.

A 23-year-old woman has had Crohn's disease since age 16. She has had a resection of the terminal ileum, and has been on and off steroids. She is currently taking infliximab and azathioprine. Her main complaints are intermittent, cramping, abdominal pain and diarrhea. She is requesting long-term disability. The gastroenterologist sees her several times a year and she is always relatively well in the clinic. She has a mild anemia, and a small bowel follow-through shows diffuse Crohn's disease in the small intestine. Where do we start with such a case?

JR: This is both a simple and complex case in that:

1. We have a disease with a well-defined pathology. In general, we know that the course is intermittent and can be severe. Bleeding and intermittent incomplete

\footnotetext{
${ }^{1}$ ASSESSMED, Mississauga; ${ }^{2}$ London Health Sciences Centre, London, Ontario

Correspondence: Dr Jack Richman, ASSESSMED, 5925 Airport Road, Suite 400, Mississauga, Ontario L4V 1W1. Telephone 905-678-2924, fax 905. 678-2939, e-mail jrichman@assessmed.com
}

Received for publication July 29, 2005. Accepted July 29, 2005 
obstructions occur causing cramps, etc; and

2. The complexity arises from the fact that each person with this disease has a varying course. For the insurer, this is a very costly claim, because this is a 23 -year-old with 42 years of insurable income-producing life ahead of her. If she is permanently disabled, it could cost the company hundreds of thousands of dollars. If she is proven disabled, the company will likely want to settle but first try to medically determine if there is work that she can do.

For the evaluating physician, one looks at the pattern of her illness and most importantly, the state of her disease in the quiescent phase. In this case, one sees that even when the disease is in a quiescent phase, the patient still has a mild anemia (which I presume is not responding to treatment) and radiologically has signs of diffuse Crohn's disease. However, before I can give an opinion as to her function, I need to determine the pattern of functioning. What I would like to know is:

a. How often does she get her cramps? Is it once a week, once a day or once every $15 \mathrm{~min}$ ?

b. How long does the cramping last? Is it $5 \mathrm{~s}$ to $10 \mathrm{~s}$, $2 \mathrm{~min}$ to $3 \mathrm{~min}$, or longer? Is just uncomfortable or does it cause her to double over?

c. What does she do to relieve it and is it successful? Have all attempts at relief failed and she has to let it run its course? How long is the course? Is it $15 \mathrm{~min}$, $2 \mathrm{~h}$ to $3 \mathrm{~h}$, or days?

d. Does medication manage the symptoms effectively?

With this important information in hand, we now need to know in detail her daily requirements at a job.

a. Is it sedentary?

b. Is it self-paced and she can take her time or does it require her to finish tasks in a time-paced manner?

c. Are there stresses in this job that would not be present in another job that aggravate the condition?

d. Does the work require her to be at the job all day, or can it be performed as she is able and at home?

e. Can the employer accommodate her?

Once we look at her functional abilities and the work requirements, we can decide if she can meet them and if she can do this on a regular basis.

It is important to have the employer write a letter stating whether they can accommodate her, as this may determine the benefits issue.

For example, I would never say she is totally disabled. Instead, I would place restrictions on her that would accommodate her condition (not the employer), such as:

1. She must have easy access to toilet facilities nearby (within 50 feet);

2. The work must be self-paced, because intermittent cramps may otherwise impede her performance; and

3. The job should allow for absences of two to three days a month on a regular basis, and perhaps longer periods intermittently, and can be made up or not as accommodated.
If the employer can accommodate her, that is great. However, it is unlikely, and therefore she would qualify for benefits because her medical condition cannot be accommodated at work.

This is important, because the decision to qualify for benefits depends on many factors and not just the doctor's note. The employer pays the premiums for the benefits and if the ill person cannot be accommodated the premiums go up. The employer then has a decision to make.

PA: What do you write on the form?

JR: On the form, or on a separate paper if there is not enough space, explain the following: "My patient has Crohn's disease, which was diagnosed by biopsy and for which she has had bowel resection surgery. It is still active radiologically as well as symptomatically, with intermittent exacerbations that leave her incapacitated for $\mathrm{xx}$ minutes (include the time for the cramps and the time for easing off) $\mathrm{xx}$ times a day and for $\mathrm{x}$ days a week. This condition prevents her from fulfilling her job requirements and her employer cannot accommodate her medical condition with the restrictions I placed on her. Therefore, she is medically disabled from performing her job. She usually responds to treatment and will take $\mathrm{x}$ weeks to return (or she is not responding to treatment and therefore will not be able to return to work for the foreseeable future). I will review her on a monthly basis."

That should qualify her, because you have met the five steps. You have:

1. Made the diagnosis;

2. Given the pathology (signs, not symptoms);

3. Defined how it is disabling for her ability to function;

4. Provided medical treatment and restrictions; and

5. Determined that she cannot be accommodated.

PA: The next scenario is a 40-year-old man with chronic hepatitis C. He is a former drug addict and alcoholic. He has failed treatment with pegylated interferon and ribavirin. He claims to be so fatigued that he needs chronic disability. He has had an ultrasound which is normal and he has slight elevations in aspartate aminotransferase and alanine aminotransferase (less than 60 $\mathrm{U} / \mathrm{L})$. How do we handle this case?

JR: This is a case in which we have a defined pathology and a diagnosis. The enzymes are above those of nonhepatitis and noncirrhotic patients, but likely not in a danger range. From a disability perspective it does not matter whether the cause is from hepatitis $\mathrm{C}$ or from alcoholic cirrhosis. The issue from the insurer's perspective is whether the person is disabled. Therefore, we must ask ourselves what is the natural history of this condition, its prognosis and what would cause the disability, if any?

Slight increases in enzymes do not cause disability and are only a sign of a liver dysfunction. The level of enzymes at low values is not prognostic of either longevity or of function. The good news is that his liver is not abnormal on the ultrasound. Perhaps a computed tomography scan is indicated. If his ultrasound and computed tomography scan are normal, these are good prognostic indicators for ongoing function.

Historically, before we found the virus that caused hepatitis $\mathrm{C}$, we called it non-A, non-B hepatitis. It was considered a relatively benign disease and most patients recovered and returned to work. Now that we have a label, one sees disability claims rising faster than for hepatitis B. Yet, on examination, there are no clin- 
ical indications of active ongoing pathology such as jaundice, increased bilirubin or ascites.

Fatigue is a part of any medical condition but is nonspecific and occurs in persons without illness. The only way to tell if there is a loss of function is through functional aerobic testing, by assessing his maximum oxygen consumption during exercise. If it is good (over the bottom 10th percentile for age and height, etc), then his fatigue is not preventing him from functioning and certainly not at a sedentary level (if he is over the 98th percentile), because this is an objective measure of deconditioning. If it is poor, then a conditioning program is in order, as mild to moderate exercise is a treatment of choice.

In the meantime, he would not qualify for benefits (except in the acute phase or if and when the disease becomes progressive).

Therefore, in this case, there is a diagnosis with defined etiological agent and pathology, but:

1. There is no active ongoing pathology;

2. There is no evidence except for self-reporting of fatigue that he is unable to function even at a sedentary level;

3. The patient claims no response to treatment; that may be true with respect to a cure but may not be true with respect to controlling/stabilizing the disease; and

4. There is no evidence that his employer cannot accommodate him.

This evidence needs to be sought out and if not present he would not medically qualify for benefits.

PA: Should chronic disability be assessed by the specialist, the family physician, a private assessment company or a government disability assessment clinic?

JR: The diagnosis, as defined by clear and present diagnostic criteria (signs), the presence of pathology and medical treatment should be under the auspices of the specialist. The family physician manages the day to day care.

If either of the two above are trained in evaluating disability then it could be assessed by them. However, that is not usually the case, and most specialist and family physicians have a conflict of interest and in their minds feel they must support a claim even if it is not justifiable. The American College of Physicians states that this unfounded support is unethical (2).

Therefore, the evaluation of the chronic disability usually falls to a physician who is trained in evaluating disability or to a medical facility or company with experience in evaluating disability. In either case, they should have no vested interest in the case or its outcome. They must use an evidence-based process and practice guidelines to make the determination.

The opinion must not only be independent of the physicianpatient relationship, but also independent of the referring source (the insurer).

PA: Is the specialist in a conflict of interest position in filling out the disability forms? If the claim is rejected the patient may be irritated at the doctor and, as is often the case, go 'doctor shopping'. In some cases, the doctor may be more sympathetic to the patient and recommend disability when it may not be appropriate (3).

JR: Although the specialist is not in a conflict of interest in filling out the disability forms, he or she should protect him- or herself by providing the following proviso, verbally or in writing, to the patient.

1. I acknowledge that you have symptoms and I have made a presumptive diagnosis, but I am unable to determine its cause or the presence of ongoing or active pathology. In the absence of this information, the insurer may determine that you do not medically qualify for benefits. In this matter, they are seeking information that neither I nor perhaps any other physician can provide to you at this time. This decision is outside my control as this is a contractual benefit in your policy.

If a doctor is more sympathetic, then in the absence of significant pathology to support a disability, the physician is putting him- or herself on the line as this type of support is considered unethical.

In its manual on ethics (2), the American College of Physicians states:

"Disability Certification: Some patients have chronic, overwhelming, or catastrophic illnesses. In these cases, society permits physicians to justify exemption from work and to legitimize other forms of financial support. In keeping with the role of patient advocate, a physician may need to help a patient who is medically disabled obtain the appropriate disability status. Disability evaluation forms should be completed factually, honestly, and promptly.

Physicians will often find themselves confronted with a patient whose problems may not fit standard definitions of disability but who nevertheless seems deserving of assistance (for example, the patient may have very limited resources or poor housing). Physicians should not distort medical information or misrepresent the patient's functional status in an attempt to help patients. Doing so jeopardizes the trustworthiness of the physician, as well as his or her ability to advocate for patients who truly meet disability or exemption criteria.

Physicians cannot and should not be expected to advise patients on the particulars of individual insurance contracts and arrangements. Patients should, however, expect their physicians to honor the rules of the insurer unless doing so would endanger the patient's health. Physicians should not collaborate with a patient or engage in efforts to defraud the insurer.

Health plans are not obliged to underwrite approaches that patients may value but that are not justifiable on clinical or theoretical scientific grounds or that are relatively cost-ineffective compared with other therapies for the same condition or other therapies offered by the health plan for other conditions."

PA: Is there a difference between private and government disability assessments?

JR: There is no difference in assessments but there is a difference in the definitions of disability and how they are applied. Often, governments rely on the attending physician to write a note, and may accept a disability even though the person may not meet the definition. This is less likely with insurance carriers. So, one may see approval for Canada Pension Plan (CPP) disability benefits but denial by a carrier. Approval by CPP does not mean that the patient met the definition of disability but could mean that the definition was not applied and the doctor's note was accepted at face value. I know that CPP is now reviewing its procedures. Although the medical examination, which includes a thorough 
history and physical examination, as well as an analysis of the documentation and the prognosis, may follow standard and evidence-based protocols, the definition of disability and how it is applied is different from one plan to another. So, one may be declared disabled in one context and not found to be disabled in another context.

PA: Some hepatitis $\mathrm{C}$ patients will request a liver biopsy to further their disability claims because evidence of cirrhosis on biopsy is more likely to be accepted than subjective symptoms. Should a patient ever undergo invasive diagnostic tests to further their claim for disability?

JR: The biopsy is for diagnostic purposes and not for ability to function. If it is clinically required for the diagnosis, then that is the only reason to perform it. If the diagnosis is not clearly made through other means then it may be indicated. Function is determined by signs of liver failure to respond to treatment; very high liver enzymes and/or bilirubin, international normalized ratio and albumin, and ascites, encephalopathy and variceal bleeding all together can give us a good picture of the extent and activity of the disease and its impact on function. The decision for a biopsy is a clinical one and not to be decided by the patient.

PA: In some diseases, patients undergo a series of athletic tests to assess their physical function, or mental function is tested by a variety of surveys. Are these recommended for gastrointestinal diseases, and can patients fake their results?

JR: All functional test results can be faked. The question is whether the tests are also designed to determine the faking. Physical and mental tests are rarely performed for gastrointestinal diseases. However, in cases where there is little or no active or ongoing pathology (such as irritable bowel syndrome) and where disability or inability to function physically or mentally through impaired cognitive function is claimed, then one must attempt to measure that loss. Because the results can be faked, built-in validity measures must be included in the testing. One looks for physiological signs of effort. For physical function, a heart rate monitor is used and if mild activity causes a significant increase in heart rate then this is an indication of deconditioning and it is likely the person does fatigue easily. But if after a few hours of activity the baseline heart rate does not increase significantly, then either the patient is better conditioned and more active than what they are reporting or they are not giving any effort. Each test is performed three times and coefficients of variation are assessed. (The three tests are added and divided by three giving a mean value. In the absence of pathology and if the patient is consistent and not faking, each of the three values usually falls within $10 \%$ of the mean.) Mental tests for cognitive function such as memory also show effort and are such that if failed it shows that they are willfully not trying, or faking.

PA: Have we created this problem by having a generous social system allowing for all of these disability pensions? Where are we heading with the budgets for these pensions and the percentage of patients trying to claim them? How do we compare with other countries?

JR: We are now getting outside the realm of medicine and you are asking me to speculate as to external causes. I will try to give my thoughts after extensive reading and experience on the subject, but should they in no way influence evaluation of the individual case. The generous social system is not an incentive for ill or injured persons to return to work. In 1986, the Menninger Foundation did a study and found that in Workers Compensation cases with similar injuries, the length of disability was highly dependent on the amount of benefits paid. This is the fault of our medical profession. When patients tell us that they want to return to work because they cannot afford to be off then, despite the severity of their medical condition we send them back to work. If they tell us they cannot return to work and want time off then despite the fact we cannot find any medical impairments we write them a note to be off. In this manner, we are not behaving as physicians but secretaries following the directions of our patients. This shows a lack of confidence in making such decisions and therefore relying on our patients to decide, our disgust at insurers and wanting to make them pay for previous transgressions, or our desire to please our patients by being their advocate at any cost. None of these reasons are justifiable. In the past, with a less generous system, economics usually made these decisions for us, because our patients who had little or no pathology could not afford to be off. Now we become responsible. We must only make medical decisions based on the medical facts. I again refer to the American College of Physicians ethics manual (2).

I must clearly state that physicians do not deny benefits. They provide the information to see whether there is medical qualification for benefits, and symptoms alone, although real, do not qualify.

On the other hand, although insurers seek medical help, they may often misunderstand the information they are given or use only parts they wish to deny benefits. This is not correct. The better a job we do in defining what is there, as in the five steps outlined above, the less likely the insurer will or can deny benefits in bona fide claims with ongoing or significant pathology (eg, Crohn's disease).

\section{REFERENCES}

1. American Medical Association. Guides to the Evaluation of Permanent Impairment, 4th edn. Chicago: American Medical Association, 1995.

2. American College of Physicians. Ethics manual. Fourth edition. Ann Intern Med 1998;128:576-94.

3. Wynia MK, Cummins DS, VanGeest JB, Wilson IB. Physician manipulation of reimbursement rules for patients: Between a rock and a hard place. JAMA 2000;283:1858-65. 


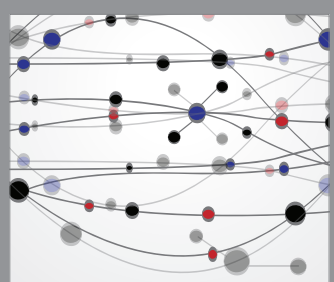

The Scientific World Journal
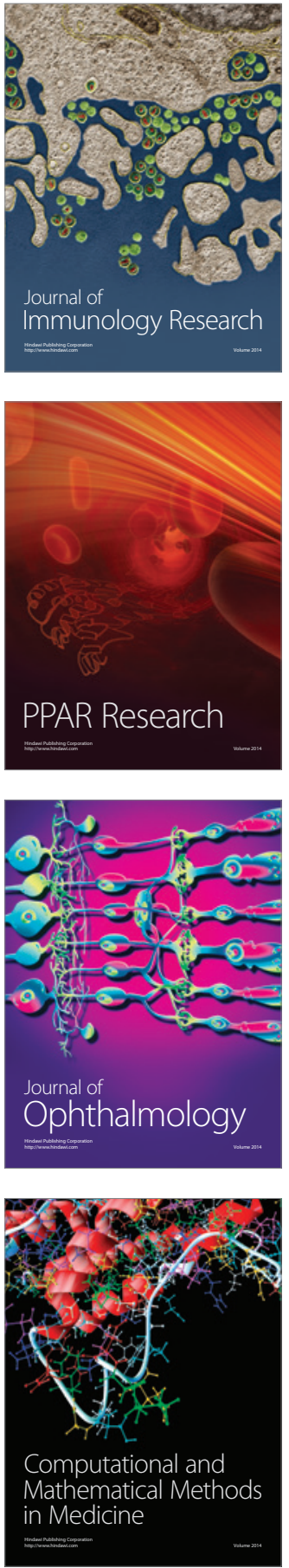

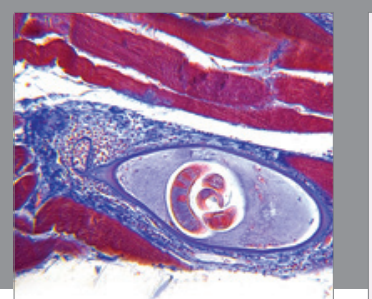

Gastroenterology Research and Practice

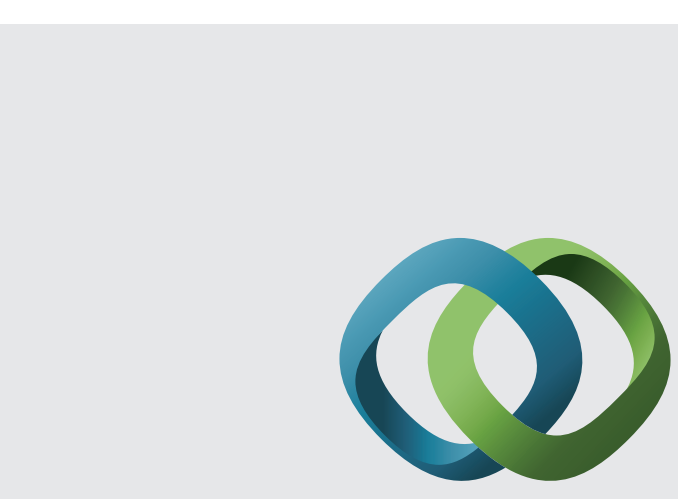

\section{Hindawi}

Submit your manuscripts at

http://www.hindawi.com
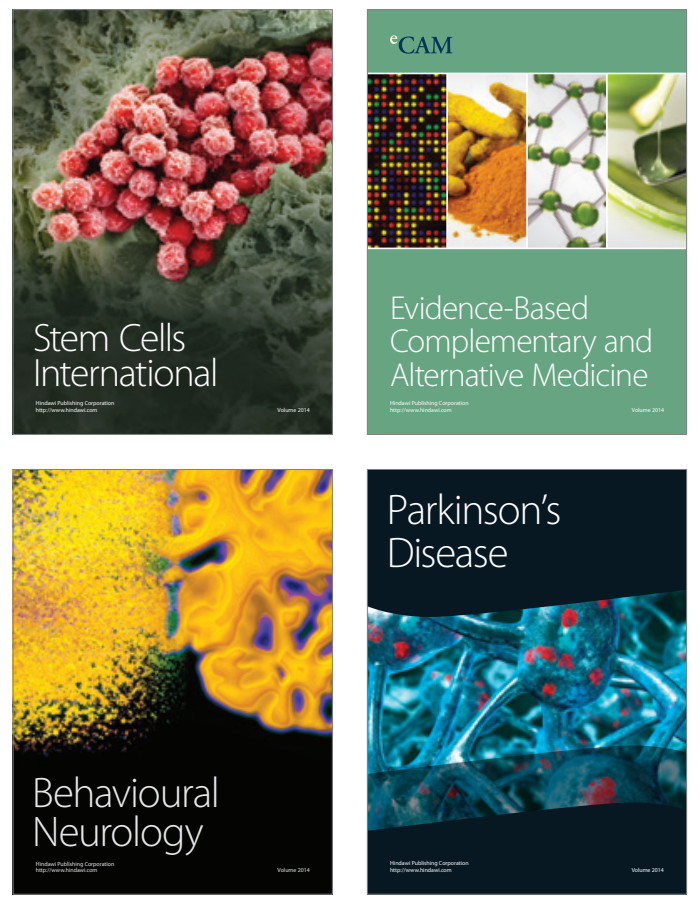
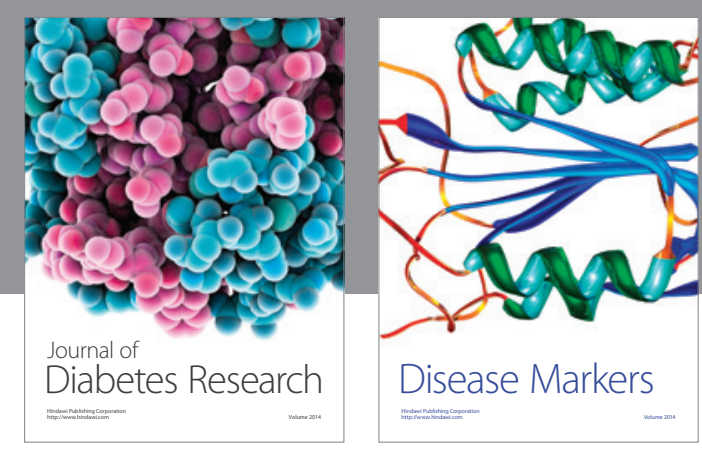

Disease Markers
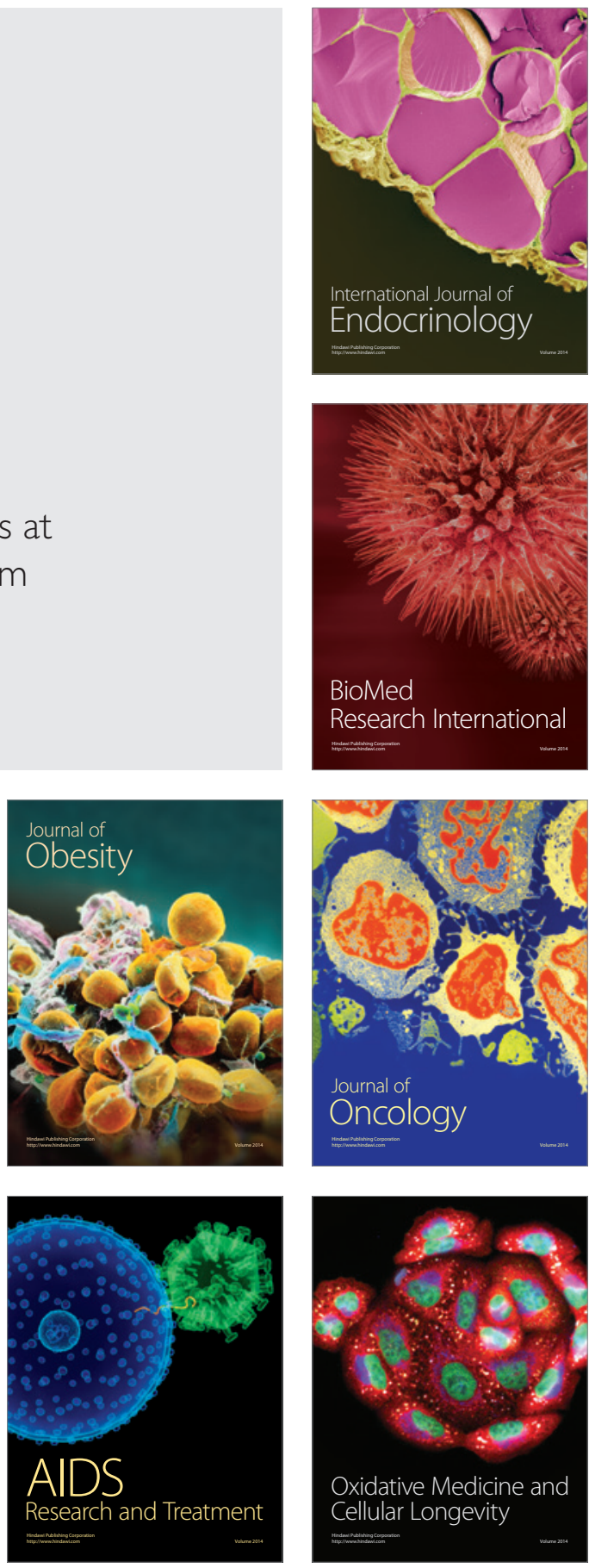